\title{
Impacts du développement des biocarburants aux États-Unis et dans I'UE sur les marchés internationaux de produits de grandes cultures
}

\author{
Yves DRONNE \\ Agneta FORSLUND \\ Alexandre GOHIN \\ Hervé GUYOMARD \\ Fabrice LEVERT
}

\author{
INRA UMR SMART, rue Adolphe Bobierre, CS \\ 61103, F-35011 Rennes-Cedex \\ <yves.dronne@rennes.inra.fr>
}

Au cours des dix dernières années, on a enregistré un très fort développement de la production et des utilisations de biocarburants dans le monde, avec un tonnage qui est passé d'environ 16 millions de tonnes en 1996 à près de 46 millions de tonnes en 2006. Bien que les marchés de biocarburants, sous forme de biodiesel et surtout de bioéthanol, représentent encore des quantités très faibles par rapport aux utilisations de carburants fossiles dans les transports, ils constituent, pour un certain nombre de produits agricoles tels que les céréales, les huiles végétales et les cultures sucrières, une forme de demande supplémentaire qui vient s'ajouter aux demandes traditionnelles pour I'alimentation humaine et animale.

Au cours des deux dernières campagnes, ce développement a été concomitant avec une hausse considérable du prix de ces matières premières qui a affecté non seulement la rentabilité de la production de ces biocarburants malgré les prix très élevés des produits pétroliers, mais aussi le coût d'approvisionnement en produits alimentaires de base comme les céréales et les huiles végétales pour de nombreuses populations du monde. Ceci a affecté également le coût de production de nombreux produits animaux, comme le lait et les viandes, tout particulièrement celui des monogastriques comme la volaille et le porc qui utilisent le plus de ces matières premières dans l'alimentation des animaux.

Cette hausse de prix est intervenue dans une conjoncture mondiale où les stocks mondiaux de nombreux produits agricoles étaient très faibles, la demande étant régulièrement depuis plusieurs campagnes supérieure à la production. Compte tenu des objectifs de remplacement de carburants fossiles très ambitieux affi-

\begin{abstract}
The development of biofuels, particularly in the United States and slightly in the European Union, has been accompanied by a strong increase in world prices for cereals and vegetable oils. The simulations using the Oleosim model in The Agriculture 2013 Foresight Study (INRA) show that under the hypothese formulated on demographic evolution and meat production in different parts of the world, world agricultural markets would be even tenser in 2015 than they were at the beginning of this century. Supplementary demand for energy purposes would have a significant additional impact on world prices, production and world trade of cereals and oilseed products, as evaluated by the Oleosim model.

Key words: biofuels, European Union, USA, world agricultural markets
\end{abstract}

chés dans certaines zones du monde telles que les États-Unis et l'Union européenne (UE) par les pouvoirs publics et des importants systèmes d'aide mis en place visant à promouvoir l'utilisation et/ou la production de ces biocarburants, il semble important d'analyser les impacts spécifiques - positifs et négatifs - que pourraient avoir à l'horizon 2015 ces programmes sur les marchés agricoles internationaux en les distinguant de tous les facteurs conjoncturels, tels que les mauvaises conditions climatiques enregistrées dans certains pays en 2006 et 2007, qui ont fortement contribué à la hausse récente des prix mondiaux des céréales et huiles végétales.

Pour effectuer cette analyse, bien que les cultures sucrières occupent une place importante en tant que substrats de production de bioéthanol, notamment à partir de la canne au Brésil et plus modestement à partir de la betterave dans I'UE, on s'est centré sur les marchés des céréales et oléagineux où jouent plus spécifiquement la concurrence au niveau des terres et des débouchés entre alimentation humaine, alimentation animale et usages énergétiques. On a utilisé le modèle OLEOSIM développé à I'INRA de Rennes pour élaborer un certain nombre de scénarios à l'horizon 2015. Ces scénarios prennent en compte les évolutions prévisionnelles des populations dans différentes zones du monde, les projections de production de viande dans ces différentes zones élaborées par les grands organismes de prévisions internationaux tels que le FAPRI et I'OCDE/FAO et deux jeux d'hypothèses sur ce que pourraient être les productions de biodiesel et de bioéthanol en 2015 aux États-Unis et dans I'UE. Dans cet article, après avoir rappelé la place des biocarburants dans l'ensemble de la demande mondiale en céréales et oléagineux au cours de l'année de référence de la modélisation (2005), on s'intéressera plus particulièrement aux résultats des simulations portant sur les évolutions de prix, de productions et de surfaces ainsi que d'échanges internationaux dans chaque scénario. Ce travail a été mené dans le cadre de la "Prospective Agriculture 2013 " mené par l'INRA entre 2006 et 2007 avec le soutien de Groupama et du Crédit Agricole.

\section{Usages alimentaires et non alimentaires des céréales et des huiles végétales à I'échelle mondiale}

Au cours de l'année 2005, environ 2,4 milliards de tonnes de céréales et oléagineux ont été produites dans le monde sur une surface d'environ 890 millions d'hectares (figure 1). Les graines oléagineuses après trituration ont fourni environ 208 millions de tonnes de tourteaux et 115 millions de tonnes d'huiles (y compris I'huile de palme) le solde de ces graines non triturées ayant été utilisé directement en alimentation humaine et en alimentation animale (environ 29 millions de tonnes). Globalement, l'alimentation humaine, sous forme de produits de base ou de produits transformés (farine, malt, amidon, etc. exprimés en équivalent grain), a utilisé environ 1,3 milliard de tonnes de ces produits dont l'essentiel correspond à des céréales (surtout blé et riz) et une autre partie importante à des huiles végétales. L'alimentation animale, avec environ 1,1 milliard de tonnes, représente un tonnage un peu plus faible que l'alimentation humaine, mais est de loin le premier débouché pour les céréa- 
les fourragères (à commencer par le maïs) et celui presque exclusif de tous les tourteaux (à commencer par celui de soja). À côté de ces deux quantités très importantes, les usages non alimentaires restent modestes (de l'ordre de 86 millions de tonnes) dont moins de 54 millions pour les biocarburants, le solde correspondant principalement à des utilisations $d^{\prime}$ 'huiles dans les savons, peintures, secteurs lipochimiques et à des utilisations d'amidon de céréales dans des secteurs industriels.

Avec une population mondiale de 6,5 milliards $d$ 'habitants en 2005, les consommations alimentaires des produits issus de céréales et d'oléagineux correspondent à une moyenne (avec naturellement des écarts considérables selon les zones géographiques considérées) de $186 \mathrm{~kg} / \mathrm{tête} / \mathrm{an}$ pour les céréales et $14 \mathrm{~kg} /$ tête/an pour les huiles végétales. Du côté de I'alimentation animale, les 1,1 milliards de tonnes de matières premières issues de céréales et d'oléagineux ont permis de produire environ 250 millions de tonnes de viandes de porc, de volaille et de bovins, soit un ratio moyen de 4,2 kilos de matières premières consommée par kilo de carcasse produit. Les céréales représentent le premier poste de consommation avec plus de $70 \%$ du total, suivi des tourteaux avec $20 \%$, le reste étant surtout constitué de coproduits de transformation des céréales tels que les corn-gluten-feeds (cgf), les dried-distillersgrains-solubles (ddgs) et les drêches de blé (ddb).

Ces bilans très simplifiés ne prennent pas en compte au niveau de l'alimentation animale I'ensemble des fourrages ni, au niveau de l'alimentation humaine, les légumes secs et tubercules qui jouent un rôle très important dans les rations alimentaires des habitants de certaines zones du monde. Au niveau des produits animaux, dans la version actuelle du modèle OLEOSIM, la production de lait n'est pas non plus prise en compte. Les résultats présentés montrent bien les nombreuses interdépendances qui existent entre les différentes filières animales et végétales, et par conséquent les multiples conséquences prévisibles $d u$ développement des biocarburants, mais ils ne permettent pas d'analyser précisément les impacts possibles, en terme de disponibilités futures en surfaces pour les grandes cultures, de certains changements dans les systèmes de production laitière (intensification ou extensification). Par ailleurs, il convient de souligner que dans toutes les simulations présentées on a supposé que la totalité des biocarburants produits en 2015 aux Etats-Unis et dans I'UE était de première génération et donc qu'à cet horizon les biocarburants de seconde génération issus en particulier de coproduits cellulosiques n'avaient pas encore un poids significatif.

\section{Les évolutions de demande mondiale à structure de consommation inchangée pour I'alimentation humaine et animale}

Dans un premier temps, nous évaluons les effets potentiels à l'horizon 2015 du développement des biocarburants de première génération sur la base de projections tendancielles de la demande mondiale alimentaire et non alimentaire en céréales et en oléagineux (huiles et tourteaux). Par construction, ces projections ignorent les ajustements via les prix sur les quantités offertes et demandées. Elles ignorent aussi les coproduits de la fabrication de biocarburants qui peuvent être utilisés en substitution d'autres ingrédients de l'alimentation animale. Dit autrement, dans un premier temps, l'attention est centrée sur une analyse "matière » hors effets prix et sans prise en compte des coproduits; ces deux éléments seront pris en compte dans un deuxième temps sur la base de simulations issues du modèle OLEOSIM.

Selon les objectifs d'ores et déjà décidés (4 \% aux États-Unis et 5,75 \% dans I'UE-25), le développement de la production d'éthanol (hors éthanol de betterave dans I'UE) représenterait, en 2015 par rapport à 2005, un besoin supplémentaire de 99 millions de tonnes de céréales (67,5 millions de tonnes aux États-Unis et 31,5 millions de tonnes dans I'UE-25). Ce tonnage peut être comparé aux besoins mondiaux additionnels en céréales pour la consommation humaine (126 millions de tonnes) et pour l'alimentation animale (121 millions de tonnes). Un renforcement des taux d'incorporation de l'éthanol à 5,25 \% aux États-Unis et $10 \%$ dans I'UE-25 correspondrait à un besoin additionnel de 154,5 millions de tonnes de céréales (98 millions de tonnes aux États-Unis et 56,5 millions de tonnes dans I'UE-25).

Ces besoins additionnels en céréales pour la fabrication d'éthanol sont à la fois modestes et importants : relativement modestes s'ils sont rapportés à la production mondiale de céréales (2005 millions de tonnes en 2005); très importants s'ils sont rapportés aux tonnages requis pour satisfaire les usages alimentaires additionnels (247 millions de tonnes en 2015 relativement à 2005) et/ou aux échanges mondiaux de céréales (126 millions de tonnes en $2005)^{1}$. En référence à ce dernier point, il importe de noter que le besoin additionnel en céréales induit par le développement de l'étha-

\footnotetext{
${ }^{1}$ Soulignons que ces chiffres diffèrent de ceux du tableau 2 dans la mesure où, à ce niveau, ils ne prennent pas en compte les ajustements prévisibles des demandes humaines et animales dus aux « effets prix »
}

nol aura lieu essentiellement dans deux pays développés exportateurs nets de céréales, alors que les besoins supplémentaires pour les utilisations humaines et animales se concentreront, pour l'essentiel, dans des pays importateurs nets de céréales, en premier lieu en Asie et en Afrique. A ce stade, le premier enseignement à retenir est donc que le développement de l'éthanol aux États-Unis et dans I'UE-25 aura, plus que vraisemblablement, un impact significatif sur les échanges mondiaux de céréales et donc, sur les prix de celles-ci.

Le même exercice reproduit pour le biodiesel suggère qu'il faudrait environ 11 millions de tonnes supplémentaires $d$ 'huiles végétales pour respecter les objectifs d'incorporation communautaires et nord-américains déjà fixés, et un peu moins de 22 millions de tonnes d'huiles végétales pour satisfaire des incorporations plus ambitieuses. Ces tonnages sont à comparer à la demande additionnelle pour la consommation humaine à l'horizon 2015, environ 9 millions de tonnes: la demande d'huiles végétales pour la fabrication de biodiesel représenterait donc entre $120 \%$ (objectifs décidés) et $240 \%$ (objectifs plus ambitieux) de l'accroissement de la demande d'huiles végétales pour la consommation humaine. Ces tonnages peuvent également être comparés à la production et/ou la consommation d'huiles végétales, aux alentours de 112 millions de tonnes en 2005, le surcroît de demande d'huiles végétales pour la fabrication de biodiesel représenterait entre $8 \%$ (objectifs décidés) et $20 \%$ (objectifs plus ambitieux) de la consommation totale d'huiles végétales de 2005.

Ces chiffres sont à mettre en relation avec la très forte dynamique de croissance de la production d'huiles. II faut également souligner que dans ces chiffres et dans le modèle OLEOSIM les 7 principales huiles végétales (palme, soja, colza, tournesol, arachide, palmiste, coprah/coco) sont prises en compte, mais il existe dans le monde un grand nombre d'autres huiles végétales, alimentaires ou non alimentaires, dont le tonnage est souvent mal cerné à travers les statistiques officielles, car largement autoconsommées, qui interviennnent également dans I'alimentation humaine de certaines zones et qui sont susceptibles d'être utilisées pour la production de biocarburants. Par ailleurs, ces chiffres ne prennent en compte ni les huiles végétales « de récupération » (huiles de fritures) ni les corps gras animaux (près de 30 millions de tonnes) qui sont, eux aussi, en partie utilisables pour la production de biodiesel. Enfin, le secteur des huiles est celui qui connaît depuis des décennies, au niveau de l'offre, la dynamique la plus forte du fait du palme et du soja, mais aussi plus récemment du colza. 
Tableau 1. Impacts en 2015 du développement des biocarburants aux États-Unis et dans I'UE-25 sur les prix mondiaux nominaux des grandes cultures, céréales et produits oléagineux (base 100 en 2005) Situations en 2015 : incorporations des biocarburants aux États-Unis et dans I'UE-25 inchangées relativement à 2005 (biocarburants inchangés); incorporations à $4 \%$ aux États-Unis et à 5,75\% dans I'UE-25 (objectifs actés); incorporations à 5,25\% aux États-Unis et à 5,75\% dans I'UE-25 (US ambitieux); incorporations à $4 \%$ aux États-Unis et à $10 \%$ dans I'UE-25 (UE ambitieuse); incorporations à 5,25\% aux États-Unis et à $10 \%$ dans I'UE-25 (double ambition US et UE)

\begin{tabular}{|lllllllll|}
\hline & & \multicolumn{2}{c}{ Graines } & \multicolumn{2}{c|}{ Huiles } & \multicolumn{2}{c|}{ Tourteaux } \\
\cline { 2 - 9 } & Blé & Maïs & Colza & Soja & Colza & Soja & Colza & Soja \\
\hline 2005 & 100 & 100 & 100 & 100 & 100 & 100 & 100 & 100 \\
2015 & & & & & & & & 116,1 \\
bioc. inch. & 111,8 & 120,3 & 106,8 & 103,6 & 101,5 & 89,6 & 111,6 \\
obj. actés & 128,3 & 129,0 & 125,7 & 110,9 & 130,8 & 126,1 & 80,3 & 101,7 \\
US amb. & 130,5 & 136,0 & 129,7 & 113,3 & 137,7 & 135,8 & 71,2 & 100,0 \\
UE amb. & 136,2 & 132,0 & 152,0 & 114,0 & 160,5 & 145,1 & 65,2 & 95,3 \\
do. amb. & 138,3 & 140,0 & 156,0 & 115,8 & 167,3 & 154,3 & 56,1 & 93,1 \\
\hline
\end{tabular}

Source : Dronne et al. (2007), modèle OLEOSIM.

Le surcroît de demande en huiles végétales à des fins énergétiques s'inscrit dans des contextes nationaux forts différents aux États-Unis et dans I'UE-25 : le premier pays est aujourd'hui (i.e., 2005) exportateur net d'huiles végétales (3,5 millions de tonnes d'équivalent huiles végétales, dont 5,1 millions de tonnes d'équivalent huile de soja) ${ }^{2}$, alors que la seconde zone est importatrice nette d'huiles végétales (10,6 millions de tonnes d'équivalent huiles végétales). Sans un considérable développement de sa production domestique de graines oléagineuses, en premier lieu de colza, l'UE-25 ne pourra satisfaire son besoin en huiles végétales à des fins énergétiques sans un recours accru aux importations de graines d'oléagineux, d'huiles végétales, voire de biodiesel. Les trois zones exportatrices nettes d'huiles végétales sont aujourd'hui I'Asie du Sud-Est (Indonésie, Malaisie et Philippines), I'Amérique du Sud (notamment, l'Argentine et le Brésil) et, dans une moindre mesure, le Canada. En résumé, le deuxième enseignement à retenir est que le développement du biodiesel dans I'UE-25 (surtout) et aux États-Unis (dans une moindre mesure) aura, plus que vraisemblablement, un impact significatif sur le commerce mondial des huiles végétales et les prix d'échange de ces dernières.

La comparaison des besoins additionnels en céréales pour l'éthanol versus en huiles végétales pour le biodiesel permet en outre de montrer que la satisfaction aux États-Unis et dans I'UE-25 des objectifs d'incorporation déjà décidés représenterait un surplus de demande proportionnellement plus important pour les huiles végétales que pour les céréales : rapporté à la consommation totale de 2005 , le besoin additionnel de céréales pour l'éthanol est d'environ $5 \%$, alors que le besoin additionnel en huiles végétales est d'environ $10 \%$; l'écart

\footnotetext{
${ }^{2}$ Les États-Unis étaient donc, en 2005, importateurs nets d'autres huiles végétales pour un total de 1,6 million de tonnes.
}

augmenterait pour des objectifs d'incorporation plus ambitieux (5,25 \% aux États-Unis et $10 \%$ dans I'UE-25): dans ce cas, le besoin additionnel en céréales pour l'éthanol est légèrement inférieur à $8 \%$, alors que le besoin additionnel en huiles végétales pour le biodiesel est d'environ $20 \%$, ces niveaux différents étant à mettre en perspective avec des dynamiques de croissance des productions qui, sur les 20 dernières années, est presque 4 fois plus importante pour les huiles (3 à $4 \%$ par an) que pour les céréales $(0,7 \%$ par an). II ne faut donc pas conclure trop hâtivement de ces ratios que les impacts des biocarburants seront nécessairement plus importants dans le cas des huiles végétales (et des tourteaux d'oléagineux) que dans le cas des céréales : en effet, le surcroît de demande en huiles végétales à des fins énergétiques représenterait de $23 \%$ (objectifs décidés) à $46 \%$ (objectifs plus ambitieux) des échanges mondiaux de 2005 d'équivalent huiles végétales, alors que le surcroît de demande en céréales pour la fabrication de bioéthanol (de 99 à 154,5 millions de tonnes) représenterait de $79 \%$ (objectifs décidés) à $132 \%$ (objectifs plus ambitieux) des échanges mondiaux de céréales. Pour aller plus loin et pleinement apprécier les impacts sur les marchés du développement des biocarburants, il est nécessaire de prendre en compte les ajustements via les prix. Ces ajustements sont décrits dans les points ci-dessous.

\section{Les implications sur les prix mondiaux des hypothèses de développement des biocarburants}

L'analyse « matière » développée ci-dessus ne prend pas en compte les ajustements des quantités offertes et demandées via les prix. Dans un second temps, le modèle OLEOSIM a donc été utilisé pour évaluer les équilibres mondiaux offre/demande, en quantités et en prix, des céréales et des oléagineux sous différentes hypothèses de développement des biocarburants aux États-Unis et/ou dans I'UE-25. Une originalité du modèle OLEOSIM est de ne pas contraindre la surface en céréales et graines oléagineuses dans les différentes zones du monde ; cette surface réagira positivement à une augmentation du produit brut moyen à I'hectare de l'agrégat grandes cultures avec des intensités différentes selon les zones, plus spécifiquement selon les disponibilités implicitement estimées en surfaces dans les différentes zones du monde (disponibilités plus fortes en Amérique du Sud que dans I'UE-25, par exemple $)^{3}$. Naturellement, les résultats présentés ci-dessous sont contingents à ces estimations implicites des terres disponibles, domaine où les incertitudes sont grandes ${ }^{4}$.

Les résultats présentés ci-après permettent donc d'apprécier les conséquences de différents niveaux de développement des biocarburants aux États-Unis et dans I'UE-25 sur les marchés mondiaux des céréales et des graines oléagineuses.

Sans développement des biocarburants, les prix mondiaux des céréales, des graines oléagineuses, des huiles végétales (à l'exception des huiles de soja et de palme) et des tourteaux d'oléagineux augmenteraient en termes nominaux par rapport à leurs niveaux de 2005, signe d'une raréfaction relative de l'offre vis-à-vis de la demande (ligne « biocarburants inchangés » du tableau 1). Les augmentations des prix mondiaux nominaux des céréales, des graines oléagineuses et des hui-

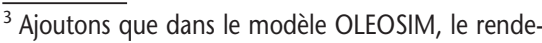
ment d'une production végétale donnée est spécifié comme une fonction d'une tendance et du produit brut à l'hectare de la production considérée.

${ }^{4}$ Ces estimations des terres disponibles sont implicites dans le modèle OLEOSIM : en pratique, elles sont représentées par les valeurs des élasticités des demandes de terres en grandes cultures céréales et oléagineux relativement au produit brut à l'hectare que ces cultures permettent de générer.
} 
Tableau 2. Impacts en 2015 du développement des biocarburants aux États-Unis et dans I'UE-25 sur les utilisations alimentaires mondiales des céréales et des huiles végétales. Les différents régimes considérés sont décrits au niveau du tableau 1 ; les huiles végétales demandées pour la production de biodiesel incluent le surcroit de demande argentine et brésilienne fixé dans tous les régimes à 2,5 millions de tonnes; unités : millions de tonnes; entre parenthèses, consommations humaines en kilogrammes par tête

\begin{tabular}{|c|c|c|c|c|c|c|c|}
\hline & \multicolumn{4}{|c|}{ Céréales } & \multicolumn{3}{|c|}{ Huiles } \\
\hline & C. humaine & A. animale & Non-alimentaire $^{a}$ & Total & C. aliment. & $\begin{array}{l}\text { Non- } \\
\text { alimentaire }^{a}\end{array}$ & Total \\
\hline Niv. 2005 & $1196,3(185,0)$ & 741,1 & 67,9 & 2005,3 & $94,1(14,55)$ & 18,5 & 112,6 \\
\hline \multicolumn{8}{|l|}{ Var. 2015} \\
\hline biocar. inch. & 108,6 & 120,9 & 00,0 & 229,5 & 9,4 & 00,0 & 09,4 \\
\hline obj. actés & 84,0 & 108,9 & 98,9 & 291,8 & 6,4 & 13,9 & 20,3 \\
\hline US amb. & 79,7 & 106,1 & 129,3 & 315,1 & 5,8 & 16,1 & 21,9 \\
\hline UE amb. & 73,3 & 104,3 & 124,0 & 301,6 & 4,9 & 22,3 & 27,2 \\
\hline double amb & 69,0 & 101,5 & 154,3 & 324,8 & 4,2 & 24,5 & 28,7 \\
\hline \multicolumn{8}{|l|}{ Niv. 2015} \\
\hline biocar. inch. & $1304,9(181,4)$ & 862,0 & 67,9 & 2234,8 & $103,5(14,39)$ & 18,5 & 122,0 \\
\hline obj. actés & $1280,3(178,4)$ & 850,0 & 67,9 & 2297,1 & $100,5(13,97)$ & 32,4 & 132,9 \\
\hline US amb. & $1276,0(177,4)$ & 847,2 & 197,2 & 2320,4 & $99,9(13,89)$ & 34,6 & 134,5 \\
\hline UE amb. & $1269,6(176,6)$ & 845,4 & 191,9 & 2306,9 & $99,0(13,76)$ & 40,8 & 139,8 \\
\hline double amb & $1265,3(176,0)$ & 842,6 & 222,2 & 2330,1 & $98,3(13,66)$ & 43,0 & 141,3 \\
\hline
\end{tabular}

Source : Dronne et al. (2007), modèle OLEOSIM.

${ }^{a}$ Ces chiffres sont différents de ceux cités précédemment dans la mesure où ils incluent, outre les matières premières utilisées pour la production de biocarburants, les utilisations pour les autres secteurs non alimentaires (environ 18 millions de tonnes pour les céréales et 14,4 pour les huiles).

les végétales seraient nettement plus importantes en cas de développement des biocarburants. En revanche, le développement des biocarburants aurait un impact négatif sur les prix mondiaux nominaux des tourteaux protéiques (ligne «objectifs d'incorporation actés » du tableau 1). Nous soulignerons la forte réactivité des cours de la graine de colza (à la hausse), des huiles de colza et de soja (à la hausse) et des tourteaux de colza (à la baisse) et, par comparaison, la moindre réactivité des cours de la graine de soja (à la hausse) et du tourteau de soja (à la baisse). La graine de soja est relativement pauvre en huile; une forte hausse du prix de l'huile de soja est donc requise pour obtenir une augmentation suffisante du prix de la graine permettant un accroissement des surfaces cultivées en soja. La baisse du prix du tourteau de soja est nettement plus faible que celle du prix du tourteau de colza : le marché du tourteau de soja étant nettement plus large que le marché du tourteau de colza, les volumes additionnels générés lors de la fabrication des biocarburants sont proportionnellement (i.e., rapportés aux tailles des marchés respectifs) significativement plus faibles dans le cas du soja que dans celui du colza. Les augmentations des cours mondiaux nominaux du blé et du maïs sont de même ampleur.

Le renforcement des objectifs d'incorporation aux États-Unis joue d'abord sur les cours du maïs et de I'huile de soja (comparaison des régimes « ambition renforcée aux Etats-Unis » et
" objectifs d'incorporation décidés aux États-Unis et dans I'UE-25 »). Mais alors que le prix du blé ne serait que marginalement affecté, les prix des diverses huiles seraient tous significativement ajustés à la hausse $(+7,7 \%$ pour l'huile de soja, $+5,3 \%$ pour I'huile de colza et $+5,0 \%$ pour I'huile de palme). La très forte baisse du prix du tourteau de colza $(-11,3 \%)$ s'explique par la concurrence avec les coproduits issus de la fabrication d'éthanol à partir de maïs.

De façon symétrique, le renforcement des objectifs d'incorporation dans I'UE-25 joue d'abord sur les cours du blé, de la graine de colza, de I'huile de colza et du tourteau de colza (comparaison des régimes « ambition renforcée dans I'UE-25 » et " objectifs d'incorporation décidés aux États-Unis et dans I'UE-25»). Cependant, les prix de toutes les huiles augmenteraient dans des proportions très importantes : $+22,7 \%$ pour I'huile de colza, $+15,1 \%$ pour l'huile de soja, $+13,6 \%$ pour l'huile de palme, et $+13,3 \%$ pour l'huile de tournesol. Dans tous les cas, les variations de prix reflètent les volatilités connues des marchés - plus élevées dans le cas des huiles que des céréales - les prix calculés restant à l'intérieur des fourchettes observées sur le passé, et en tout état de cause se situent à I'horizon 2015 à des niveaux très inférieurs à ceux observés en 2007 et au début de 2008.

\section{Les implications sur les utilisations alimentaires de céréales et $\mathbf{d}$ 'huiles végétales des hypothèses de développement des biocarburants}

Un impact négatif, mais modéré, sur les utilisations alimentaires de céréales et d'huiles végétales en 2015

Les variations des cours mondiaux nominaux des céréales et des oléagineux sont le fruit de la confrontation des offres et des demandes (humaine, animale et non alimentaire). Dans chaque régime, les utilisations de céréales et d'huiles végétales pour la fabrication de biocarburants sont fixées (déterminées par hypothèse). C'est donc au niveau des utilisations alimentaires, humaines et animales, que se font les ajustements à la baisse de la demande induits par les augmentations des prix des céréales et des huiles végétales ${ }^{5}$.

Le tableau 2 met ainsi en lumière la concurrence potentielle entre usages non alimentaires et alimentaires des céréales et des huiles végé-

\footnotetext{
${ }^{5}$ La présentation linéaire adoptée ici (impact du développement des biocarburants sur les prix, puis les demandes, enfin les offres) ne doit pas occulter le fait que les ajustements des prix et des quantités, demandées et offertes, sont simultanés.
} 
Tableau 3. Impacts en 2015 du développement des biocarburants aux États-Unis et dans I'UE-25 sur les productions, les surfaces et les rendements en céréales et en oléagineux. Les différents régimes considérés sont décrits au niveau du tableau 3.1 ; niveaux 2005 en millions de tonnes (productions), millions d'hectares (surfaces) et tonnes par hectare (rendements)

\begin{tabular}{|c|c|c|c|c|c|c|}
\hline & \multicolumn{3}{|c|}{ Céréales } & \multicolumn{3}{|c|}{ Huiles } \\
\hline & Production & Surface & Rendement & Production & Surface & Rendement \\
\hline Niv. 2005 & 2005,3 & 672,7 & 2,98 & 425 & 216,8 & 1,96 \\
\hline \multicolumn{7}{|l|}{ Var. 2015} \\
\hline biocar. inch. & $11,4 \%$ & $4,5 \%$ & $6,8 \%$ & $10,3 \%$ & $4,5 \%$ & $5,8 \%$ \\
\hline obj. actés & $14,5 \%$ & $6,2 \%$ & $8,3 \%$ & $14,6 \%$ & $8,0 \%$ & $6,6 \%$ \\
\hline US amb. & $15,7 \%$ & $6,8 \%$ & $8,9 \%$ & $15,2 \%$ & $8,4 \%$ & $6,8 \%$ \\
\hline UE amb. & $15,0 \%$ & $6,6 \%$ & $8,4 \%$ & $17,7 \%$ & $10,4 \%$ & $7,3 \%$ \\
\hline double amb. & $16,2 \%$ & $7,2 \%$ & $9,0 \%$ & $18,3 \%$ & $10,9 \%$ & $7,4 \%$ \\
\hline
\end{tabular}

Source : Dronne et al. (2007), modèle OLEOSIM.

tales, plus spécifiquement l'effet négatif du développement des biocarburants sur la croissance des utilisations alimentaires de céréales et $d^{\prime}$ huiles végétales. Cet impact n'est pas négligeable, aussi bien pour les céréales que pour les huiles végétales: les tonnages additionnels de céréales (respectivement, d'huiles végétales) pour l'alimentation humaine et animale passeraient ainsi d'un indice 100 dans un régime de taux incorporation aux États-Unis et dans I'UE-25 constants aux niveaux de 2005 à un indice 74,3 (respectivement, 44,6 ) dans un régime $d$ 'incorporation des biocarburants à hauteur de 5,25\% aux États-Unis et de $10 \%$ dans I'UE-25. Rapportés aux consommations humaine et animale, les impacts à la baisse seraient néanmoins modérés: la consommation alimentaire de céréales (respectivement, d'huiles végétales) passerait d'un indice 100 en 2015 dans un régime de taux incorporation constants aux niveaux de 2005 (indice qui correspond à 2167 millions de tonnes de céréales et 103,5 millions de tonnes d'huiles végétales) à un indice 97,3 (respectivement, 95,0) dans un régime de renforcement des objectifs d'incorporation aux États-Unis et dans I'UE. En tenant compte de l'évolution démographique à l'horizon 2015, les consommations humaines par tête de céréales baisseraient de 181,4 kilogrammes (taux d'incorporation constants aux niveaux de 2005) à 176,0 kilogrammes (renforcement des objectifs d'incorporation aux ÉtatsUnis et dans I'UE-25); pour les deux mêmes régimes, les consommations humaines par tête d'huiles végétales baisseraient de 14,39 à 13,66 kilogrammes ${ }^{6}$.

II ne faut pas conclure de l'analyse précédente que la situation alimentaire mondiale se dégra-

\footnotetext{
${ }^{6}$ Sur la décennie 2005-15, I'hypothèse retenue est une augmentation de la population mondiale de $11,1 \%$, très inégalement répartie selon les zones : de manière générale, la croissance démographique serait plus importante en Afrique, en Asie et Amérique du Sud (Dronne et al., 2007). Par suite, les évolutions des consommations par tête de céréales et
}

dera à hauteur de la baisse observée des consommations humaines par tête de céréales et d'huiles végétales. Le bilan alimentaire mondial complet doit également prendre en compte, d'une part les autres produits végétaux utilisés pour I'alimentation humaine, notamment les légumes secs et les tubercules (autres végétaux non pris en compte dans le modèle OLEOSIM), d'autre part les différents produits animaux (produits animaux pris en compte, de façon simplifiée, dans le modèle et qui augmentent sur la décennie 2005-15). Cette remarque ne doit cependant pas occulter le résultat majeur mis en exergue par les chiffres du tableau 2, à savoir : I'effet contraire du développement des biocarburants aux États-Unis et dans I'UE-25 sur les consommations alimentaires de céréales et d'huiles végétales.

\section{Les implications sur les surfaces et productions de céréales et d'huiles végétales des hypothèses de développement des biocarburants}

A l'horizon 2015, une demande de biocarburants compatible avec l'évolution tendancielle des rendements sous réserve d'augmentations des surfaces en céréales et graines oléagineuses comprises entre 5 et $10 \%$

Augmentations des rendements et des surfaces emblavées se combinent pour satisfaire les besoins additionnels en céréales et en huiles végétales requis par le développement des biocarburants tout en satisfaisant l'augmentation des usages alimentaires. Ceci est détaillé dans le tableau 3 qui met en lumière les points suivants : (i) les augmentations des rendements et

d'oléagineux seront différentes selon les zones géographiques. des surfaces sont d'autant plus élevées que le développement des biocarburants est important, (ii) l'augmentation requise des rendements est compatible avec la croissance tendancielle observée sur les deux dernières décennies, et (iii) l'augmentation requise des surfaces est loin d'être négligeable: dans le régime où les objectifs d'incorporation sont renforcés dans les deux zones, la surface en céréales (respectivement, graines oléagineuses) devrait augmenter de 7,2\% (respectivement, 10,9\%) par rapport à 2005, et cet effet à la marge extensive contribuerait pour $44 \%$ (respectivement, $60 \%$ ) à l'accroissement de la production mondiale céréalière (respectivement, oléagineuse).

Une lecture alternative des pourcentages d'augmentation des surfaces présentés dans le tableau 3 est d'interpréter ceux-ci comme les accroissements exogènes additionnels de rendements requis pour des surfaces en céréales et en oléagineux supposées (maintenues) constantes aux niveaux de 2005. Selon cette lecture, les accroissements de rendements seraient nettement plus élevés, au maximum $16,2 \%$ pour les céréales et $18,3 \%$ pour les graines oléagineuses. Ces accroissements devraient être obtenus en dix années.

Les chiffres du tableau 3 correspondent à des moyennes à l'échelle de la planète; ils masquent des évolutions contrastées dans les diverses zones du monde, principalement en fonction des réserves de terres mobilisables pour la culture de céréales et d'oléagineux. Le tableau 4 illustre cette diversité sur l'exemple des surfaces. Sans développement des biocarburants dans I'UE-25 et aux États-Unis, la demande additionnelle en céréales et en produits oléagineux nécessiterait d'augmenter les surfaces cultivées en céréales et en oléagineux de plus de $20 \%$ en Amérique du Sud (Argentine et Brésil). Les évolutions des surfaces seraient nettement plus faibles dans toutes les autres zones du monde, avec en outre des évolutions contrastées des surfaces céréalières (augmentation) et des surfaces oléagineuses 
Tableau 4. Impacts en 2015 du développement des biocarburants aux États-Unis et dans I'UE-25 sur les surfaces en céréales et en oléagineux dans diverses zones du monde. Les différents régimes considérés sont décrits au niveau du tableau 3.1 ; niveaux 2005 en millions d'hectares (base 100 entre parenthèses) ; l'Asie du sud-est regroupe I'Indonésie, la Malaisie et les Philippines

\begin{tabular}{|c|c|c|c|c|c|c|c|}
\hline \multicolumn{8}{|c|}{ Panel a. Surfaces en céréales } \\
\hline & $\begin{array}{c}\text { Argentine et } \\
\text { Brésil }\end{array}$ & UE-25 & États-Unis & Canada & Chine & $\begin{array}{c}\text { Asie du Sud- } \\
\text { Est }\end{array}$ & $\begin{array}{c}\text { Afrique } \\
\text { subsaharienne }\end{array}$ \\
\hline Niv. 2005 & 28126 & 51324 & 56538 & 16394 & 80767 & 22379 & 83590 \\
\hline Indice 2015 & (100) & (100) & (100) & (100) & (100) & (100) & $(100)$ \\
\hline biocar. inch. & 125,7 & 101,8 & 106,8 & 103,8 & 100,9 & 104,0 & 101,4 \\
\hline obj. actés & 138,3 & 101,6 & 108,5 & 96,7 & 100,9 & 109,3 & 101,6 \\
\hline US amb. & 143,0 & 101,7 & 110,3 & 96,4 & 101,0 & 110,2 & 101,9 \\
\hline UE amb. & 142,5 & 100,7 & 109,4 & 91,8 & 101,1 & 111,4 & 101,6 \\
\hline double amb. & 147,0 & 100,8 & 111,1 & 91,4 & 101,0 & 112,3 & 101,9 \\
\hline \multicolumn{8}{|c|}{ Panel b. Surfaces en oléagineux } \\
\hline & $\begin{array}{c}\text { Argentine et } \\
\text { Brésil }\end{array}$ & UE-25 & États-Unis & Canada & Chine & $\begin{array}{c}\text { Asie du Sud- } \\
\text { Est }\end{array}$ & $\begin{array}{c}\text { Afrique } \\
\text { subsaharienne }\end{array}$ \\
\hline Niv. 2005 & 41152 & 7517 & 36587 & 6527 & 27613 & 13846 & 13951 \\
\hline Indice 2015 & $(100)$ & $(100)$ & $(100)$ & $(100)$ & $(100)$ & (100) & $(100)$ \\
\hline biocar. inch. & 122,3 & 95,8 & 96,1 & 92,7 & 99,5 & 103,9 & 100,3 \\
\hline obj. actés & 126,2 & 103,9 & 96,0 & 111,7 & 100,2 & 109,0 & 102,2 \\
\hline US amb. & 128,0 & 105,1 & 94,5 & 112,9 & 100,3 & 109,8 & 101,9 \\
\hline UE amb. & 128,5 & 114,4 & 95,8 & 125,3 & 100,5 & 111,0 & 103,7 \\
\hline double amb. & 130,4 & 115,5 & 94,3 & 126,6 & 100,9 & 111,9 & 103,3 \\
\hline
\end{tabular}

Source : Dronne et al. (2007), modèle OLEOSIM.

(diminution) dans I'UE-25, aux États-Unis et au Canada. Le développement des biocarburants dans I'UE-25 et/ou aux États-Unis aurait un impact positif très important sur les surfaces en céréales et en oléagineux en Amérique du Sud : dans l'hypothèse d'un fort développement dans les deux zones, les surfaces céréalières augmenteraient de près de 17 points de pourcentage $(147,0 / 125,7)$ et celles en oléagineux d'un peu plus de 8 points de pourcentage (en hectares, les augmentations seraient d'un même ordre de grandeur, aux alentours de 13 millions d'hectares dans les deux cas). La promotion des biocarburants dans I'UE-25 et/ou aux États-Unis aurait un impact légèrement positif sur les surfaces céréalières aux Êtats-Unis et un impact légèrement négatif sur les surfaces oléagineuses dans ce pays. Par contraste, l'impact sur les surfaces oléagineuses serait positif dans I'UE-25 et au Canada (dans ce pays, au détriment de la sole céréalière qui diminuerait de plusieurs points de pourcentage $)^{7}$. ̀̀ l'exception de l'Amérique latine et de la zone dite Asie du sud-est qui regroupe l'Indonésie, la Malaisie et les Philippines, l'impact du développement des biocarburants dans I'UE-25 et aux

\footnotetext{
${ }^{7}$ L'impact positif sur les surfaces canadiennes en oléagineux serait plus important dans le cas d'un développement des biocarburants dans I'UE-25 (demande d'huiles végétales pour le biodiesel) versus aux États-Unis.
}

États-Unis sur les surfaces en céréales et en oléagineux dans les autres zones du monde serait moindre ; ceci est particulièrement le cas dans les pays de l'Afrique subsaharienne. Néanmoins, l'impact serait toujours positif sous l'effet des augmentations des prix des céréales et des huiles végétales.

\section{Les implications sur la structure des échanges de céréales, d'huiles végétales et de coproduits des hypothèses de développement des biocarburants}

\subsection{Un impact important sur} la structure des échanges de céréales, d'huiles végétales et de coproduits

Les évolutions contrastées des offres et des demandes de céréales, d'huiles végétales, de tourteaux d'oléagineux et de coproduits de transformation des céréales et des oléagineux en biocarburants, ont pour effet de considérablement modifier la physionomie des échanges mondiaux de ces différents produits. Dans l'année de base, 2005, les États-Unis sont, et de loin, la première zone exportatrice nette de céréales (pour un peu plus de 83 millions de tonnes, dont un peu plus de 54 millions de tonnes de maïs) ; les autres zones exportatrices nettes sont l'Argentine et le Brésil, le Canada et I'UE-25. Au niveau des huiles végétales (plus précisément des huiles végétales et graines oléagineuses exprimées en équivalent huile), les zones exportatrices nettes en 2005 sont I'Asie du sud-est (pour environ 26 millions de tonnes, dont 22 millions de tonnes d'huile de palme), I'Argentine et le Brésil (pour environ 15 millions de tonnes, dont près de 14 millions de tonnes d'huile de soja), les États-Unis (pour un peu plus de 3,5 millions de tonnes, dont un peu plus de 5 millions de tonnes d'huile de soja) et le Canada (pour un peu plus de 3,2 millions de tonnes, dont 3,2 millions de tonnes d'huile de colza); I'UE-25 et la Chine sont deux zones importatrices nettes pour des ordres de grandeur voisins, aux alentours de 11 millions de tonnes (huiles de palme et de soja).

Sans développement des biocarburants aux États-Unis et dans I'UE-25, les exportations nettes de céréales des zones déjà excédentaires en 2005 augmenteraient, y compris dans I'UE$25:+30,6$ millions de tonnes en Argentine et au Brésil, $+24,9$ millions de tonnes aux ÉtatsUnis, $+10,8$ millions de tonnes dans I'UE-25 (accroissement dans I'UE-25 qui serait plus important sous I'hypothèse additionnelle d'une suppression du gel des terres obligatoire) et $+6,2$ millions de tonnes au Canada; la Chine et l'Afrique subsaharienne verraient leur position se détériorer avec des accroissements 


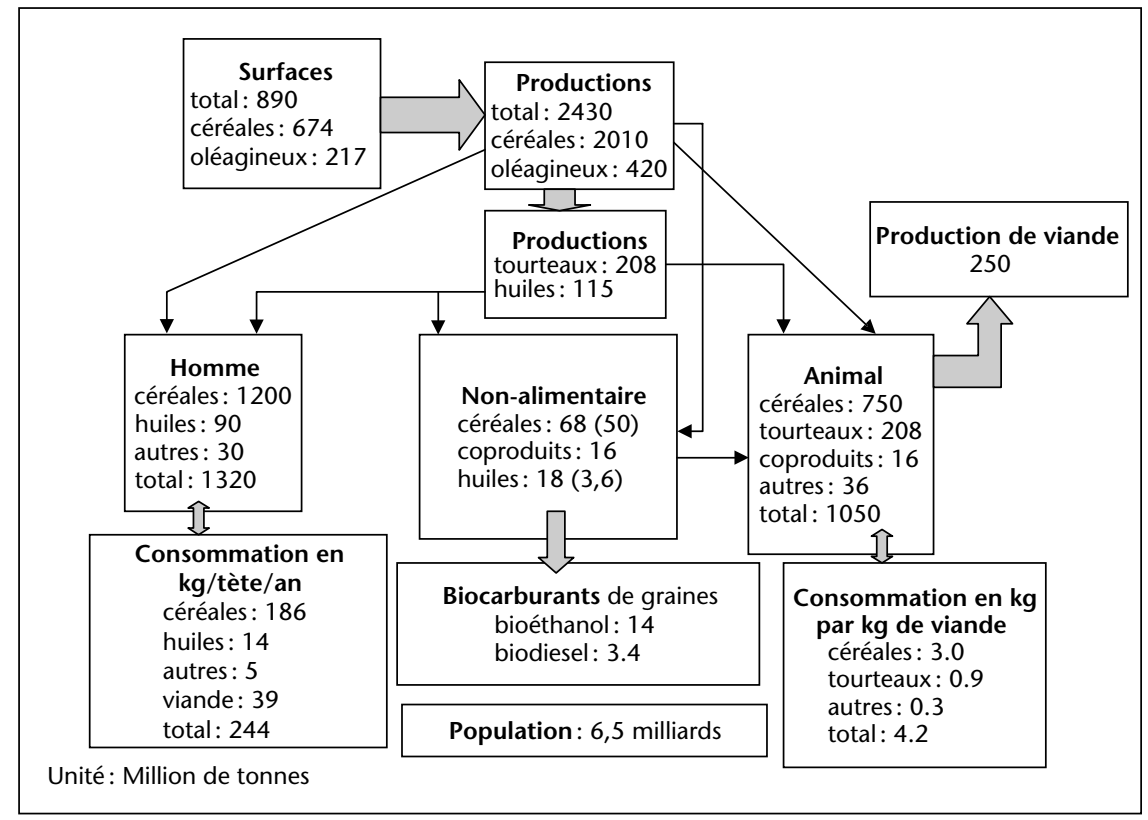

Figure 1. La structure du marché mondial en 2005.

des importations nettes de 26,3 millions de tonnes et 19,8 millions de tonnes, respectivement. Les évolutions seraient plus limitées sur les marchés des huiles, avec une augmentation des exportations nettes en Argentine et au Brésil, ainsi qu'en Asie du sud-est, et une détérioration de la balance commerciale dans toutes les autres zones du monde; on notera néanmoins la stabilité des importations d'huiles végétales dans I'UE-25. Enfin, sur les marchés des tourteaux protéiques, on contrastera la dégradation du solde négatif chinois (qui se détériore de 8,5 millions de tonnes), avec I'augmentation des exportations argentines et brésiliennes (+ 23,1 millions de tonnes).

Le développement des biocarburants dans I'UE-25 aboutit à une réduction des exportations communautaires nettes de céréales, réduction d'autant plus forte que le taux d'incorporation des biocarburants dans I'UE-25 est élevé : dans l'hypothèse d'un développement ambitieux $(10 \%)$, la réduction est telle que l'UE-25 serait importatrice nette de céréales, et pour des tonnages très importants (plus de 35 millions de tonnes). Les importations communautaires d'huiles végétales augmenteraient, alors que les importations communautaires de tourteaux protéiques diminueraient. De façon plus générale, sur le marché mondial des céréales, I'UE-25 serait importatrice nette pour des tonnages importants, les États-Unis réduiraient très fortement leurs exportations, alors que l'Amérique latine augmenterait très substantiellement ses exportations; sur le marché des huiles végétales, I'UE-25 et les ÉtatsUnis augmenteraient fortement leurs importations au bénéfice de l'Amérique latine, et de I'Asie du sud-est (figure 1).

\section{Conclusion}

Comme le montrent les simulations précédentes, le développement de la production de biocarburants aux États-Unis et dans I'UE au cours des prochaines années risque d'entraîner une pression accrue sur les marchés des céréales et des huiles végétales et donc une hausse des prix de ces produits par rapport à ce que l'on avait enregistré en 2005. II faut toutefois noter que les deux années suivantes, 2006 et 2007, sous l'effet du développement des biocarburants, mais aussi d'un ensemble de facteurs conjoncturels, les prix internationaux ont déjà connu des hausses importantes. Ainsi pour le maïs, on est passé en Fob Gulf de $100 \$$ /tonne en 2005 à 124 en 2006 et 170 en 2007. De même pour l'huile de colza en Dutch Fob ex Mill on est passé de 669 \$/tonne en 2005 à 794 en 2006 et 969 en 2007. Les évolutions de prix entre 2005 et 2007, notamment pour ces deux produits, sont très importantes et même supérieures à ce qui a été calculé avec le modèle OLEOSIM pour I'horizon 2015. En fait ces résultats ne sont pas contradictoires dans la mesure où dans la simulation on a retenu une hypothèse de production de biocarburants certes plus importante que ce qui a été enregistré en 2007, mais, en sens inverse, on a retenu une hypothèse d'évolution tendancielle des rendements et de progression des surfaces dans le monde qui vient pour une large part amoindrir les effets du choc de demande. Ceci montre bien que les impacts des biocarburants sur les marchés agricoles mondiaux dépendront non seulement des niveaux de production qui seront atteints, mais aussi de leur rythme de développement. Les ajustements de surfaces entre cultures et les progressions des surfaces cultivées par rapport notamment aux prairies et pâturages, de même que les effets des gains de productivité (notamment sur les rendements agricoles, mais aussi sur les indices de consommation alimentaire en productions animales) ne peuvent s'exprimer que progressivement. Les effets risquent donc d'être très différents selon que des objectifs identiques seront atteints en 2015 ou en 2020.

Globalement, de la même façon que le développement des biocarburants a un impact sur les matières premières agricoles, l'évolution des prix des matières premières joue et continuera à jouer sur la rentabilité des biocarburants. Le développement de ces derniers dépendra alors de plus en plus des incitations des politiques nationales mises en œuvre en vue de promouvoir leur production et leur utilisation.

Les résultats présentés dans cet article sont naturellement contingents aux hypothèses qui ont été formulées et ce travail se poursuit actuellement dans plusieurs directions. II s'agit d'abord d'améliorer la désagrégation des zones géographiques dans le modèle, $d$ 'introduire explicitement dans celui-ci les offres et demandes de produits de plantes sucrières et d'affiner le choix de matrices d'élasticités. II s'agit ensuite dans la définition de nouveaux scénarios à l'horizon 2015 voire 2020 $d^{\prime}$ une part de prendre en compte les objectifs de production de biocarburants de première génération de certaines zones du monde autres que I'UE et les États-Unis, telles que la Chine, I'Inde, le Brésil, et aussi introduire des hypothèses sur les productions de biocarburants de seconde génération, certaines des cultures impliquées étant susceptibles de venir concurrencer des cultures alimentaires traditionnelles. 